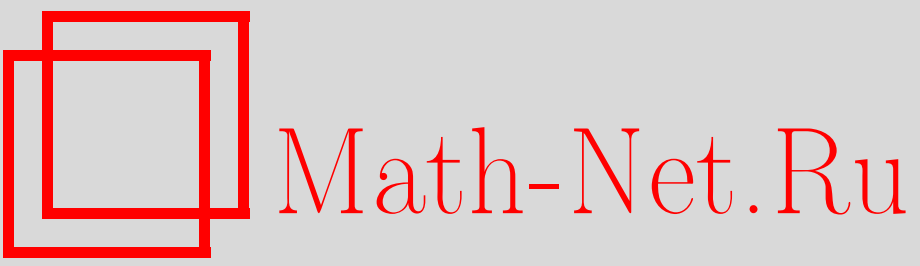

Н. П. Долбилин, М. А. Штанько, М. И. Штогрин, О неизгибаемости полиэдральных сфер с четноугольными гранями, УМН, 1996, том 51, выпуск 3, 197-198

DOI: https://doi.org/10.4213/rm982

Использование Общероссийского математического портала Math-Net.Ru подразумевает, что вы прочитали и согласны с пользовательским соглашением

http://www.mathnet.ru/rus/agreement

Параметры загрузки:

IP : 54.80 .97 .219

26 апреля 2023 г., 18:15:05 


\title{
О НЕИЗГИБАЕМОСТИ ПОЛИЭДРАЛЬНЫХ СФЕР С ЧЕТНОУГОЛЬНЫМИ ГРАНЯМИ
}

\author{
Н. П. ДолБИлин, М. А. ШтАньКО, М. И. ШтоГРИн
}

В этой заметке мы продолжаем изучение вопроса о неизгибаемости невыпуклых многогранников в $\mathbf{E}^{3}$, начатое в работах [2]-[4]. Метод графа изгибания, описанный в этих работах, находит здесь дальнейшее развитие. С основными понятиями, результатами и проблемами теории изгибания поверхностей можно познакомиться в сборнике [1].

Рассмотрим полиэдральную сферу $S^{2}$, составленную из евклидовых многоугольников, и ее вложение $f: S^{2} \rightarrow \mathbf{E}^{3}$, при котором каждая грань сферы $S^{2}$ отображается изометрично в плоский многоугольник, расположенньй в пространстве.

Допустим, что вложенная сфера $f\left(S^{2}\right)$ допускает непрерывное изгибание $\varphi$, в процессе которого каждая грань остается плоской. В процессе изгибания $\varphi$ хотя бы один двугранный угол, т.е. угол между плоскостями двух смежных граней, изменяется. Будем называть всякое ребро изменяющегося при $\varphi$ двугранного угла, отмеченным. Рассмотрим изгибание $\varphi$ и будем предполагать его настолько малым, что вложенная сфера при изгибании $\varphi$ остается по-прежнему вложенной. Множество всех отмеченных ребер образует граф Г. Ребра графа - это отмеченные ребра сферы $f\left(S^{2}\right)$, а вершины графа - это вершины отмеченных ребер и только они. Напомним свойства графа отмеченных ребер.

Г1. Пусть изгибание ч существует, тогда граф Г не пуст.

Г2. Граф Г разбивает сферу $f\left(S^{2}\right)$ на несколько компонент сязности. Каждое отмеченное ребро разделяет две различные компоненты, т.е. является границей двух граней полиәдральной сферы $f\left(S^{2}\right)$, принадлежащих разным компонентам.

Г3. В каждой вершине графа должно встречаться не менее двух ребер.

Г4. Если в вериине графа Г сходятся только два его ребра, то они лежат на прямой.

Г5. В вериине графа Г не может сходиться ровно 3 его ребра.

По той же схеме, как это делалось в [3], [4], перейдем от графа отмеченных ребер к другому, так называемому графу изгибания $\Gamma_{\varphi}$. Вершинами графа изгибания $\Gamma_{\varphi}$ являются лишь те вершины графа $\Gamma$, в которых сходится не менее 4 отмеченных ребер. Рассмотрим произвольное ребро $e$ из графа Г и выделим те отмеченные ребра, которые можно соединить с $e$ реберным путем, проходящим через вершины графа Г степени 2 . В силу ограниченности сферы $f\left(S^{2}\right)$ и свойства Г4 такие ребра образуют прямолинейный отрезок, который является по соглашению ребром графа изгибания $\Gamma_{\varphi}$.

$\Gamma_{\varphi} 1$. Все ребра графа $\Gamma_{\varphi}$ суть прямолинейнье отрезки, состоящие из одного или нескольких ребер, входящих в граф Г.

$\Gamma_{\varphi}$ 2. Никакие два ребра графа $\Gamma_{\varphi}$ не имеют два общих конца.

Это вытекает из того, что $f\left(S^{2}\right)$ есть вложение в $\mathbb{R}^{3}$. Таким образом, в графе изгибания нет двуреберных циклов.

$\Gamma_{\varphi}$ 3. В каждой вериине графа $\Gamma_{\varphi}$ сходится не менее 4 ребер.

$\Gamma_{\varphi} 4$. Граф $\Gamma_{\varphi}$ разбивает сферу на области, среди которых по крайней мере один треугольник (под треугольником понимается область, граница которой состоит из трех и только трех ребер графа $\left.\Gamma_{\varphi}\right)$.

Точно так же, как и в [2]-[4], графф изгибания $\Gamma \varphi$ разбивает многогранную поверхность $f\left(S^{2}\right)$ на связные области, среди которых есть по крайней мере восемь таких, что граница каждой из них есть треугольник в графе $\Gamma_{\varphi}$ ("условие 8 треугольников").

Теорема 1. Пусть полиэдральная сфера $S^{2}$ содержит лишь четноугольнье грани и все ее вериины - строго положительной кривизны. Пусть $f$ - вложение сферы $S^{2}$ в $\mathbf{E}^{3}$, которое локально изометрично и линейно на каждой грани. Тогда сфера $f\left(S^{2}\right)$ не допускает изгибания, при котором все грани остаются плоскими.

Работа выполнена при частичной поддержке Российского фонда фундаментальных исследований (грант № 96-01-00166) и Немецкого научно-исследовательского общества (DFG). 
ДокАЗАТЕЛЬСтво теоремы проведем от противного. Пусть $f\left(S^{2}\right)$ допускает непрерывное изгибание $\varphi$, и пусть $\Gamma_{\varphi}$ - соответствующий граф̆ изгибания.

В силу строгой положительности кривизны вершин граф изгибания $\Gamma_{\varphi}$ совпадает с графом отмеченных ребер Г. Действительн, так как каждая вершина $v$ полиэдра $S^{2}$ имеет строго положительную кривизну, то в ней не могут сходиться лишь два отмеченных ребра (свойство Г4). Так как в графе $\Gamma$ нет двойных вершин, то каждое ребро графа $Г$ является ребром графа $\Gamma \varphi$ и обратно. Таким образом, граф $\Gamma$ совпадает с $\Gamma \varphi$. В графе $\Gamma=\Gamma \varphi$ существуют, по крайней мере, восемь треугольников. Каждый треугольник составлен из трех и только трех ребер графа Г. Такой треугольник является одномерным циклом на сфере $f\left(S^{2}\right)$ и, следовательно, гомологичным 0 по модулю 2. Заметим однако, что на ориентируемой поверхности с четноугольными гранями любой реберный цикл, гомологичный нулю по модулю 2 , как нетрудно доказать, содержит четное число ребер (см., например, [4]). Полученное противоречие доказывает теорему 1.

СлЕдСТВИЕ. Каждое невыпуклое вложение поверхности выпуклого многогранника в евклидово пространство $\mathbf{E}^{3}$ с четноугольными гранями неизгибаемо.

Теорема 1 допускает следующее усиление.

ПредлОЖение 1. Пусть полиэдральная сфера $S^{2}$ содержит не более семи нечетноугольных граней и все ее вериины - строго положительной кривизны. Пусть $f$ вложение сферы $S^{2}$ в $\mathbf{E}^{3}$, которое локально изометрично и линейно на каждой грани. Тогда сфера $f\left(S^{2}\right)$ не допускает изгибания, при котором все грани остаются плоскими.

ДокАЗАтЕльство. Допустим, что данная поверхность изгибаема. Среди областей, на которые граф̆ $\Gamma_{\varphi}$ разбивает поверхность $f\left(S^{2}\right)$, имеется по крайней мере восемь областей, обозначим их через $F_{i}$, которые ограничены треугольниками $\Delta_{i}$ (условие восьми треугольников). Так как все вершины на $S^{2}$ имеют строго положительную кривизну, то графы $\Gamma=\Gamma \varphi$ и каждая сторона треугольника $\Delta_{i}$ состоит из одного и только одного ребра многогранной поверхности $f\left(S^{2}\right)$.

Воспользуемся следуюшим фактом: если односвязная область состоит лишь из четноугольных граней, то граница этой области есть также четноугольник. Тогда, если бы полиэдральная сфера $S^{2}$ имела бы менее восьми нечетноугольных граней, то по крайней мере одна из областей $F_{1}, \ldots, F_{8}$ состояла бы только из четноугольных граней и, следовательно, ограничивающий ее контур не мог бы быть треугольным. Отсюда следует предложение 1.

В заключение заметим, что изложенные здесь результаты верны также и для значительно более широкого класса отображений $f$ полиэдральной сферы $S^{2}$. А именно, по-прежнему предполагая отображение $f$ локально изометричным и линейным на каждой грани, будем требовать от отображения $f$ лишь то, что оно является погружением реберного остова полиэдра $S^{2}$ в $\mathbf{E}^{3}$. Другими словами, результаты остаются верными, даже если допускается, что образ $f\left(S^{2}\right)$ может самопересекаться, но при этом образы ребер, исходящих из одной вершины $v$ сферы $S^{2}$, пересекаются, как и их прообразы, в одной точке - в образе вершины $f(v)$.

\section{СПИСОК ЛИТЕРАТУРЫ}

[1] Исследования по метрической теории поверхностей. Сборник статей. Сер. математика. Новое в зарубежной науке. Т. 18. М.: Мир, 1980. [2] Dolbilin N. P., Stan'ko M. А., Stogrin M.I. Rigidity of Quadrillages of the Sphere // Международная конференция по геометрии "в целом". Черкассы, 1995. С. 22-23. [3] Долбилин Н.П., Штанько М.А., Штогрин М.И. Неизгибаемость квадрильяжа сферы // ДАН. 1996 (в печати). [4] Долбилин Н.П., Штанько М.А., Штогрин М.И. // УМН. 1996. Т. 51. № 2. С. 157-158. [5] Долбилин Н.П., Штанько М.А., Штогрин М.И. // Труды МИАН. 1991. T. 196. C. $66-85$.

Математический институт

им. В. А. Стеклова РАН

Принято редколлегией 18.04.1996 